\title{
Apprentissage plurilingue et théâtre. Le chemin fructueux de la pluridisciplinarité
}

\section{Arianna Berenice De Sanctis}

\section{(2) OpenEdition}

Édition électronique

URL : http://journals.openedition.org/esp/2370

DOI : 10.4000/esp.2370

ISSN : 2532-0319

Éditeur

Centre d'Information sur l'Éducation Bilingue et Plurilingue

Édition imprimée

Date de publication : 1 juin 2018

Pagination : $57-70$

ISSN : 1127-266X

\section{Référence électronique}

Arianna Berenice De Sanctis, « Apprentissage plurilingue et théâtre. Le chemin fructueux de la pluridisciplinarité », Éducation et sociétés plurilingues [En ligne], 44 | 2018, mis en ligne le 08 février 2019, consulté le 30 avril 2019. URL : http://journals.openedition.org/esp/2370 ; DOI : 10.4000/ esp.2370 


\section{APPRENTISSAGE PLURILINGUE ET THÉÂTRE. LE CHEMIN FRUCTUEUX DE LA PLURIDISGIPLINARITÉ}

\section{Arianna Berenice DE SANGTis}

La pratica teatrale permette di esplorare corporalmente le strutture linguistiche e facilita lo sviluppo di nuove strategie di comunicazione. L'apprendimento di una o più lingue attraverso un approccio ludico giova al tempo stesso al rafforzamento delle strutture grammaticali e all'ampliamento del vocabolario. Inoltre, trasmettere competenze linguistiche nell'ambito di un laboratorio di teatro riduce quegli ostacoli nell'espressione orale che possono sorgere in un contesto scolastico 0 universitario.

Parole chiavi: Apprendimento, lingue stramiere, teatro, incarnazione

Acting makes it possible to explore linguistic structures through the body, as well as to develop new communication strategies. Learning one or more languages through a recreational approach, benefits both a reinforcement of the grammatical structures and a broadening of the vocabulary. In addition, the change in setting where training takes place helps to reduce the constraints that often limit oral expression in a school or university context.

Keyzerds: Learning activity, foreign languages, theatre, embodiment.

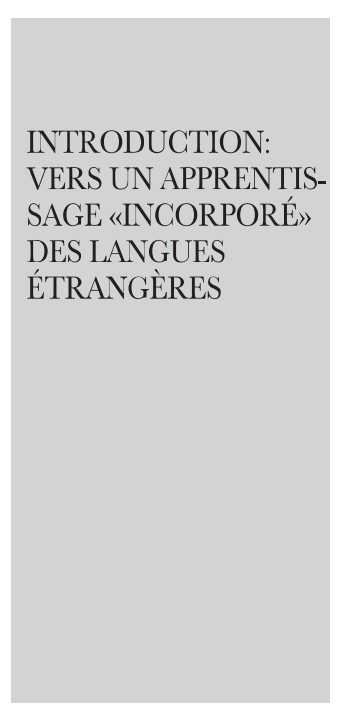

\section{L'acquisition du langage est une fonction humaine qui s'exerce comme une technique du corps (Cohen 1969).}

La pratique théâtrale facilite l'exploration par le corps des structures linguistiques ainsi que le développement de nouvelles stratégies de communication. Par ce biais, l'apprentissage d'une ou plusieurs langues, par une approche ludique, profite en même temps d'un renforcement des structures grammaticales et d'un élargissement du vocabulaire. En outre, le changement du cadre dans lequel la formation se déroule peut favoriser un allégement des contraintes qui limitent l'expression orale dans un contexte scolaire ou universitaire.

L'application de différents exercices issus du théâtre, du mime corporel, de la danse et du masque, combinée avec un parcours d'écriture dramatique individuelle et collective, conduit les participants à créer de courtes partitions personnelles (à la fois physiques, musicales et textuelles). Celles-ci peuvent faire l'objet d'une restitution à la fin de l'atelier.

Actuellement ATER dans le département de théâtre à l'Université Paul Valéry Montpellier 3, j’ai enseigné la langue et la culture italien- 
Apprentissage plurilingue et théâtre. Le chemin fructueux de la pluridisciplinarité

A. B. De SANCtis ne pendant dix ans en Île de France. Depuis 2015, je donne des ateliers de théâtre pour apprendre la langue italienne et française dans les départements d'études romanes des universités de Tübingen et de Stuttgart ainsi qu'à la Pädagogische Hochschule de Karlsruhe.

Ma formation dans le théâtre s'est déroulée d'abord en Italie puis en France où j'ai suivi une formation intensive de mime corporel pendant deux ans et demi (sous la direction de Luis Torreão et de Thomas Leabhart, Paris). En qualité de mime, d'actrice, de metteure en scène et de pédagogue, j'ai créé des spectacles dans les compagnies Hippocampe (Paris), Le Noud (Paris) et La Théâtreria (Saint-Denis/Buenos Aires), entre 2007 et 2015.

En ce qui concerne l'enseignement des langues (italien, français), j’ai exercé en France et en Italie dans des milieux différents tels que des écoles publiques et privées et des associations. Ceci m'a permis d'adapter et de développer des techniques pédagogiques à des publics très variés et de mieux comprendre le rôle du corps et de l'action dans les processus d'apprentissage. En effet, comme l'expliquent Alain Berthoz et Jean-Luc Petit «ancrer le langage dans l'action, l'inter-animation, l'échange de l'acte, loin de rabaisser la langue restitue à celleci un fondement de sa puissance» (Berthoz, Petit 2006: 60).

Au cours des trois dernières années (2015-2018), grâce au soutien et à l'ouverture d'esprit des collègues allemands, j'ai mis en place différents ateliers de théâtre sous forme de courtes sessions intensives ou de séances se déroulant durant tout un semestre universitaire. L'objectif principal étant, aussi bien pour les enseignants que pour les étudiants, de développer une approche pragmatique des langues et de privilégier l'expression orale.

En effet, si dans les cursus universitaires outre-Rhin, la grammaire, la phonétique, la civilisation et la littérature sont abordées comme des matières essentielles dans la formation, les cours de conversation et les activités extra-scolaires restent rares. La majorité des étudiants qui décide de participer aux ateliers déclare le faire dans le but de pratiquer la langue de manière plus spontanée, afin de renforcer le vocabulaire acquis, de mieux s'approprier le registre quotidien et les expressions issues du langage courant.

Les ateliers s'adressent à un groupe de cinq à vingt étudiants qui ont une bonne connaissance de la grammaire et des structures de la langue étrangère (niveau CECRL B1/B2).

Dans certains cursus, les inscrits au département d'études romanes suivent une double formation en études italiennes et françaises. Dans ce cas, un atelier bilingue peut être proposé, durant lequel l'intervenant donne des indications dans les deux langues, permettant ainsi aux participants de percevoir leurs structures communes, mais aussi 
Apprentissage plurilingue et théâtre. Le chemin fructueux de la pluridisciplinarité

A. B. De SANCTIS de repérer les «faux amis». Tout au long de l'atelier, la démarche bilingue se déroule suivant une méthode immersive: les étudiants sont invités à s'adresser à l'enseignant, à échanger avec leurs camarades et à réaliser les exercices dans l'une des deux langues étrangères.

Afin d'être à l'aise dans le travail physique, les participants portent une tenue souple, et contribuent à la composition de la partition finale en apprenant par cœur, en amont de l'atelier, une strophe d'une chanson de leur choix dans une des langues de l'atelier. Le parcours d'exercices se déroule de préférence dans une salle de cours vide ou dotée de chaises et de tables amovibles et s'inspire de multiples sources.

Pour l'exploration des relations avec les objets, la méthode utilisée est celle du mime corporel; en ce qui concerne l'élaboration de la partition physique, textuelle et vocale, l'apport principal vient des stages animés par l'Odin Teatret (Holstebro, Danemark), et des workshops que j'ai eu l'occasion de suivre tout au long de ma formation théorique et pratique dans les études théâtrales (aussi bien en Europe qu'en Amérique latine).

D'autres matériaux de création ont été adaptés à partir des performances et des expériences conduites entre 2007 et 2015 avec Ariel Divone, Fernanda Docampo et Gabriele Sofia, membres fondateurs de la compagnie La Théâtreria. Selon sa durée et ses objectifs, l'atelier peut aborder 1) les techniques de respiration, de placement et de projection de la voix (articulation et exploration de différentes intonations et rythmes), 2) le développement de la concentration et de l'écoute, 3) la lecture et l'interprétation de textes courts (niveau progressif de Al à B2) ainsi que la rédaction spontanée de textes brefs, 5) la posture, le déplacement dans l'espace scénique, 6) le mime corporel, la danse, l'improvisation et les jeux de rôle, 7) l'utilisation et la manipulation d'objets, 9) Les techniques de montage et de mise en scène.

Le parcours n'est, de préférence, jamais fixé au préalable, car le niveau des exercices doit être adapté en fonction du public, de la durée de l'atelier et des relations à l'intérieur du groupe. C'est pour cela que, à la fin de chaque séance, il est conseillé de consacrer un temps à l'échange entre les participants et avec l'intervenant afin qu'émergent les souhaits, les craintes et les difficultés, et que l'on puisse rapidement rééquilibrer les tâches à accomplir pour faire en sorte que l'atelier se déroule dans la manière le plus harmonieuse possible. Quelques étudiants ayant participé aux ateliers de théâtre pour l'apprentissage des langues étrangères animés à l'université de Stuttgart et de Tübingen signalent que l'un des obstacles majeurs rencontré consiste en l'angoisse de devoir parler et chanter en public, car ils n'ont pas l'habitude de s'exposer de cette manière dans le cadre d'un 
Apprentissage plurilingue et théâtre. Le chemin fructueux de la pluridisciplinarité

A. B. De SANCtis cours de langue en milieu universitaire. Généralement, ils sont rassurés quand on leur explique que cette prise de parole n'est pas l'objet d'une évaluation selon des critères grammaticaux et syntaxiques. Une crainte ultérieure qui apparait est celle de ne pas être à la hauteur de produire un échange verbal immédiat et spontané et, par conséquent, de perdre la face à l'égard des camarades (et de l'enseignant). À ce propos, il est nécessaire de préciser dès le début de l'atelier que l'implication de chacun et le soutien et la bienveillance de tous sont des éléments indispensables pour la réussite de l'atelier car une attitude positive contribue à créer un environnement de travail agréable et facilite l'acquisition de nouvelles compétences. Dans certains rapports rédigés à la fin de ces ateliers, les étudiants ont témoigné:

«Devo dire che non ho mai frequentato un corso di questo tipo perché avevo sempre paura di fare un figuraccia, ma con questo corso ho imparato che a volte è molto importante fare le cose che normalmente non si osa fare». «Je dois avouer que je n'ai jamais fréquenté un cours de ce genre car j'avais toujours peur de me ridiculiser, mais en participant à cet atelier j'ai appris que parfois il est très important de faire des choses que normalement nous n'osons pas faire.» (Sophie, Stuttgart, janvier 2017)

«Ho trovato molto utile che (l'insegnante) ha partecipato all'inizio (di ogni esercizio) per aiutarci a vincere la nostra paura e ad avvicinarci alla materia. Così abbiamo potuto sviluppare e realizzare le nostre proprie idee.» «J'ai trouvé très utile le fait que l'enseignante ait participé au début de chaque exercice pour nous aider à dépasser notre crainte et à nous approcher du thème. Nous avons ainsi pu développer et réaliser nos propres idées.» Alexa, Stuttgart, février 2017).

«Durante la prima seduta si doveva parlare liberamente molto spesso, ciò può essere molto stressante e difficile per alcune persone perché in quel momento non si conosce ancora bene il gruppo.» «Pendant la première séance on devait parler librement très souvent et cela peut être très stressant et difficile pour certaines personnes, car à ce moment-là on ne connaît pas bien le groupe.» (Floria, Tübingen, juillet 2017).

«ll corso non era difficile o faticoso da seguire ma il tempo troppo limitato non ha permesso di sviluppare i diversi aspetti teatrali. Personalmente, avrei voluto approfondire il fatto di parlare più liberamente, specialmente in italiano. Ciononostante, è stata un'esperienza formidabile dato che non avevo mai fatto teatro in una lingua straniera e il piccolo gruppo ha creato un ambiente di lavoro molto rilassato.» «Le cours n'était pas difficile ou fatiguant à suivre, mais la durée limité n'a pas permis de développer les différents aspects du travail théâtral. Personnellement, j'aurais voulu parler plus librement, spécialement en italien. 
Apprentissage plurilingue et théâtre. Le chemin fructueux de la pluridisciplinarité

A. B. De SANCtis

L'ACTIVATION DES CODES DE OMMUNICATION CORPORELS
Cependant, cela a été une expérience formidable étant donnée que je n'avais jamais fait du théâtre en langue étrangère et que le petit groupe a crée une atmosphère de travail très détendue.» (Linus, Stuttgart, janvier 2017).

Plusieurs enchaînements d'exercices peuvent être réalisés selon la durée de l'atelier et le nombre des participants. De manière générale, le même exercice est répété deux fois, les tâches à accomplir se complexifiant à chaque étape. Dans un premier temps, les étudiants exécutent un exercice de base, puis quelques minutes plus tard, ils sont encouragés à le répéter en y introduisant une petite variation. Ainsi, le niveau de difficulté est légèrement augmenté de manière à accompagner l'étudiant vers une progression graduelle tout en veillant à garder une approche sereine de son apprentissage.

Ensuite, lors de l'accomplissement de l'exercice suivant, la transition de la simplification à la complexification intervient de nouveau et le même procédé est appliqué pour les épreuves qui suivent. Tout au long de la séquence, les participants avancent par spirales ascendantes, entre l'acquis et l'«acquérir». Agissant de cette manière, le risque de découragement chez l'étudiant diminue tandis que sa disposition à apprendre augmente. À ce propos, Gregory Bateson (1977: 201-202) explique:

«Si néanmoins nous faisons subir à un même sujet une série d'expériences d'apprentissage similaires, nous découvrirons qu'à chaque expérience qui s'ajoute, le gradient d'apprentissage primaire augmente, ce qui veut dire que le sujet apprend de plus en plus vite. C'est ce changement progressif du taux d'apprentissage primaire que nous appellerons 'apprentissage secondaire'».

Lors de la première séance, l'intervenant expose brièvement son parcours et les modalités de déroulement de l'atelier. Afin de faciliter la concentration et d'encourager un lâcher-prise, il est nécessaire de mettre en place un chemin d'apaisement codifié, éloigné des codes de communication d'un cours frontal. Pour ce faire, dès le début de l'atelier, les participants limitent la place du verbal lors de la prospection du terrain physique, exception faite pour les exercices qui prévoient une combinaison des deux dimensions.

La séquence commence toujours par des échauffements où, la prise de parole tressée à un mouvement, aide les étudiants à se présenter par le corps et avec leur imaginaire. Disposés en cercle, l'un après l'autre, les étudiants réalisent un geste de leur choix accompagné de la prononciation de leur prénom à haute voix. L'action physique est à proposer en même temps que l'«action» vocale et une ampleur du geste et de la voix sont à rechercher dès la première exécution de l'exercice.

Lors d'un deuxième tour de cercle, tout le monde répète le geste et le 
Apprentissage plurilingue et théâtre. Le chemin fructueux de la pluridisciplinarité

A. B. De SANCtis son présentés par chaque participant, le but étant de les reproduire de la manière la plus fidèle possible. Enfin, dans un troisième tour, les mouvements et les prénoms s'enchaînent les uns après les autres en créant une sorte de chorégraphie chantée, commune au groupe entier. De cette manière, la première personne du cercle prononce son prénom et reproduit le mouvement esquissé, la deuxième les répète et enchaîne avec sa propre suggestion, le troisième reproduit les deux propositions des camarades qui le précèdent suivi de la sienne, et ainsi de suite.

L'exercice demande une très grande concentration et opère à la fois sur le renforcement de la mémoire, de l'écoute et de la cohésion de groupe, tout en gardant l'aspect ludique favorisé par le cadre artistique dans lequel il se déploie.

La pratique de l'enseignement des gestes pour consolider la formulation orale et incorporer les structures linguistiques est désormais courante. À ce propos Marion Tellier souligne que:

«Enfin, les gestes des apprenants peuvent avoir une fonction de méta-commentaire, c'est-à-dire qu'ils commentent l'interaction en cours. Ils sont utilisés pour maintenir la conversation et pour résoudre des problèmes dus au déficit de la compétence langagière de l'apprenant. Ainsi, donc, outre une double fonction purement linguistique (aide lexicale et grammaticale), le geste a une fonction plus phatique» (Tellier 2012: 78).

L'entraînement se poursuit par des exercices fondés sur l'activation des différentes parties du corps et qui aident à échauffer les articulations, à prendre conscience des tensions internes et à visualiser les différents segments du corps, tout en explorant leurs limites et leurs connexions. Les participants se disposent en cercle et commencent à mouvoir une partie du corps, en procédant du haut vers le bas (la tête, le cou, une épaule, puis le torse, le bassin, etc.) dans les différentes directions (en haut, en bas, à droite, à gauche, en effectuant des rotations...).

Après une courte démonstration de l'intervenant, ce sont les participants qui jouent le rôle d'instructeur les uns après les autres en donnant à leurs camarades des suggestions, en activant le vocabulaire relatif au corps humain enrichi de l'emploi de connecteurs spatiaux.

De cette manière, à l'aide de leur imaginaire, ils peuvent diriger les actions en utilisant des adverbes ou des locutions adverbiales tels que «devant, derrière, au-dessus, en-dessous, plus haut, d'un côté...de l'autre côté», de groupes nominaux précédés d'une proposition comme «à gauche, à droite, au fond, à l'arrière, etc.», ainsi que d'autres indicateurs de lieu «en face de la porte, à côté de la fenêtre, sur la table, contre la cloison, etc.». L'entrelacement du vocabulaire du corps humain et des connecteurs spatiaux peut faire surgir des 
Apprentissage plurilingue et théâtre. Le chemin fructueux de la pluridisciplinarité

A. B. De SAnctis indications de ce genre: «Bougez la tête d'un côté... puis de l'autre côté, montez les coudes en direction du plafond, poussez l'épaule droite vers le sol, déplacez le genou gauche vers la fenêtre, etc.».

Une séquence portant sur l'exploration de différentes manières de marcher dans l'espace peut compléter cette première partie de l'atelier. L'animateur invite les participants à se déplacer librement dans la salle, en faisant attention à se répartir dans l'espace de manière équilibrée.

Les indications incitent les étudiants à explorer des rythmes différents (accélérer, ralentir, s'arrêter d'un coup) et à des niveaux différents (sauter, toucher le sol, ramper), à imaginer de déambuler sur un sol qui varie en qualité et matière (feu, glace, eau, métal, boue, cailloux, etc.) ou encore à trouver des formes différentes d'appui (partie extérieure ou intérieure du pied, talons ou orteils, etc.). Puis, chaque étudiant à son tour prend le relai et développe les consignes de l'intervenant en donnant des suggestions à partir de son propre imaginaire dans le but d'aiguiser les cinq sens:

«Marchez sur le sable chaud du désert», «nagez dans l'océan froid au clair de la lune», «déplacez-vous dans l'espace en apesanteur comme les cosmonautes», «avancez et reculez dans la salle en imaginant que vous êtes entourés par une matière gélatineuse et parfumée», etc.

Une évolution possible de cet exercice consiste à donner quatre consignes telles que «marcher», «s'arrêter», «sauter» et «toucher le sol», puis de les inverser, en gardant les mêmes formules, mais en inversant leurs significations. De cette manière, quand l'intervenant prononce le mot «marchez» les étudiants doivent s'arrêter, tandis que quand il donne la consigne «arrêtez-vous» ils doivent reprendre la marche.

La même règle est appliquée quand il dit «sautez» ou «touchez le sol». L'exercice peut être réalisé une dernière fois en faisant coïncider de nouveau les consignes et les actions. Par ce biais, la concentration et la réactivité des participants sont renforcées grâce à un processus alterné d'association et dissociation du système verbal et moteur.

L'exploration dans l'espace se poursuit par la danse des impulsions. Les participants choisissent un point du corps et se meuvent dans la salle en animant le déplacement à partir de ce point. Ils visualisent au fur et à mesure différents points et alternent une impulsion après l'autre afin de varier leur démarche et leur posture. L'intervenant les guide et, au bout de quelques minutes, leur propose de le remplacer dans la direction. L'objectif est de faire prendre conscience de plusieurs types et qualités de mouvement qui peuvent inspirer la création d'un solo de danse ou d'un corps spécifique d'un personnage issu d'un 
Apprentissage plurilingue et théâtre. Le chemin fructueux de la pluridisciplinarité

A. B. De SANCtis

CONSTRUIRE LE SENS À L'AIDE DE L'AUTRE: LE TRAVAIL EN PETITS GROUPES matériel dramatique donné (texte, image, chanson, etc.).

Cette première partie de l'atelier permet à l'étudiant de s'échauffer, de se familiariser avec l'espace, de réveiller en douceur le vocabulaire acquis et d'atteindre une qualité d'écoute de son corps qui lui permettra de poursuivre le travail de création en solo, en duo ou en groupe. L'enchaînement des exercices est pensé de sorte que le contact physique entre les participants se développe progressivement, pour respecter le rapport à l'intimité de tout un chacun, cette «dimension cachée» que E. Hall définit comme étant «celle du territoire de tout être vivant, animal ou humain, de l'espace nécessaire à son équilibre» (Hall 2014, $4{ }^{\text {ème }}$ de couverture), dans laquelle l'aspect culturel joue un rôle essentiel.

Dans la deuxième partie de l'atelier, les participants se mettent deux par deux, l'un manipule l'autre en guise de marionnette. En effleurant le camarade, le premier soulève et laisse tomber délicatement d'abord ses doigts, puis sa main, son avant-bras, son coude et son bras. Le deuxième (les yeux fermés ou ouverts) essaye de lâcher complètement son poids et de s'abandonner au partenaire. Le contact physique est léger mais cependant essentiel, il permet de percevoir l'autre, d'apprendre à lui faire confiance, à construire un processus de création commun.

Un exercice inspiré de la technique de manipulation du Bunraku (marionnettes japonaises) permet de prolonger cette proposition, tout en stimulant davantage l'écoute et en perfectionnant la coordination. Les étudiants se répartissent en groupes de quatre, l'un d'eux ferme les yeux et joue le rôle de marionnette, les trois autres se placent derrière lui et agissent comme des marionnettistes. Un premier, accroupi au sol, manipule les deux jambes, un deuxième le bras droit et la tête, un troisième le bras gauche. L'équipe essaie tout d'abord de trouver un terrain d'entente en accomplissant, une après l'autre, des actions minimes (petits déplacements en lenteur) pour ne pas confondre l'«acteur-marionnette» avec une trop grande variété de consignes. Les trois manipulateurs changent de rôle pour expérimenter les différentes positions et les sensations qui y sont liées. Puis un parmi eux (celui qui agit sur la tête et le bras gauche) choisit une action à faire accomplir à la marionnette (s'assoir sur une chaise au fond de la salle, aller prendre une bouteille d'eau sur la table, ouvrir la porte, etc.). Le «chef manipulateur» communique par le corps son intention aux deux autres en transmettant en douceur quelques signaux au camarade manipulé. Il esquisse quelques mouvements pour suggérer une trajectoire que les collègues suivent en tâchant de réagir assez rapidement afin de faciliter une mise en mouvement organique de la «marionnette». Les trois 
Apprentissage plurilingue et théâtre. Le chemin fructueux de la pluridisciplinarité

A. B. De SANCtis agissent ensemble et coordonnent leurs mouvements pour compléter et faire évoluer dans l'espace l'action impulsée par le chef manipulateur. Une fois que chaque groupe marionnette-manipulateurs a effectué quelques déplacements dans la salle et qu'il est rodé, une épreuve supplémentaire s'ajoute. Plusieurs quatuors se rencontrent et font effectuer à leurs «marionnettes» des actions simples (se saluer, se serrer dans les bras, s'assoir l'un à côté de l'autre, etc.). Lors de l'exécution de la première phase de l'exercice «Bunraku», les mots sont limités car les participants affinent en priorité leur capacité d'écoute et renforcent leur coordination tacite. Cependant, dans la deuxième étape, l'élément vocal fait son apparition. Lorsque deux quatuors se rencontrent dans la salle, les «acteurs-marionnettes» peuvent improviser des salutations, échanger des mots, des dialogues et mêmes des bribes de chansons.

Cette activation de la sphère verbal apparaît à la fin d'un parcours muet qui demande beaucoup d'énergie et de concentration, et elle est vécue par les participants plutôt comme une étape de relaxation que comme un obstacle supplémentaire.

De fait, la manipulation à trois exige une focalisation sur les impulsions, ce qui conduit à une condensation et à une réduction du mouvement dans son essence et oblige à une précision et une écoute de l'autre constantes et continues. D'un côté, ce processus peut engendrer un sentiment de frustration chez l'exécutant car il limite sa liberté d'action en faveur de celle du groupe. De l'autre côté, les difficultés posées par ce travail physique minutieux peuvent déclencher le mécanisme de désinhibition cognitive, qui comme le précise Joëlle Aden:

«[...] Nous permet de masquer les informations sensorielles, émotionnelles ou intellectuelles qui ne sont pas pertinentes ou qui sont superflues pour les objectifs que nous nous fixons. [...] Les filtres cognitifs permettent au sujet de mobiliser ce qu'il sait sans prendre en compte les traitements mentaux d'arrièreplan» (Aden 2016: 109).

Ainsi, en se concentrant davantage sur les tâches à accomplir dans son parcours physique (tâches qui se complexifient à chaque nouvelle étape), l'étudiant peut temporairement faire abstraction des difficultés soulevées par le maniement des langues étrangères. L'introduction graduelle de mots l'aide à aborder confortablement l'épreuve orale lorsque cela lui est demandé. La superposition progressive de phrases simples, dont lui-même évalue la portée, facilite le repérage d'un vocabulaire précis et réduit le trac et la peur de ne pas être à l'hauteur dans la performance orale.

Pour faciliter une prise de parole plus fluide et plus intense, tout en gardant l'apport favorablement contraignant imposé par le chemin corporel, dans la troisième partic de l'atelier les participants se mesu- 
Apprentissage plurilingue et théâtre. Le chemin fructueux de la pluridisciplinarité

A. B. De SAnctis rent à l'expression orale par des brèves improvisations.

Ils racontent en quelques phrases et à tour de rôle ce qu'ils ont fait le matin avant d'arriver à l'atelier (ou le soir précédent). Ils disposent de quelques minutes pour réfléchir et organiser la structure de leur récit en y ajoutant trois ou quatre actions physiques proposées par les camarades lors de l'exercice de présentation réalisé au tout début de la séance.

Une variation consiste à placer les étudiants assis sur une chaise, et à leur demander de résumer en quelques phrases une histoire qu'ils connaissent très bien (qui appartient à leur imaginaire, à leur vécu, ou bien tirée de la littérature mondiale). Derrière la chaise, un partenaire débout, placé de dos ou de profil, invente une petite danse improvisée. Il suit la musicalité des paroles du récit, en réagissant par son corps aux sons et aux pauses de la langue.

Cet exercice qui vise à construire à deux une histoire-dansée dans l'immédiateté, demande une certaine souplesse et une capacité d'entreprendre. Sa forme ludique permet aux élèves de ne pas créer des attentes trop ambitieuses pour eux-mêmes et leur laisse la possibilité de choisir le sujet et le rythme de leur intervention. Comme le montrent Berthoz et Petit, ce processus de mise en commun reflète mieux en général le fonctionnement des relations humaines et sous-tend la communication:

Seule l'action partagée peut unir deux subjectivités dans un même flux d'interactions constituantes. [...] Co-constituer le monde par une action partagée est la modalité première et fondamentale du pouvoir d'interagir avec autrui (Berthoz, Petit 2006: 250, 246).

Dans un souci d'alternance et pour permettre aux étudiants de se reposer des épreuves où ils ont été davantage sollicités dans la production orale, pour leur permettre aussi d'élargir la palette de nuances de leur expression corporelle, il est envisageable d'introduire dans cette troisième étape une activité sur les masques neutres en papier.

Quelques masques sont distribués à la moitié des participants, tandis que les autres jouent le regard curieux et attentif du public. Il est demandé au premier groupe d'observer le masque posé sur la paume de la main, puis de le placer sur le visage en l'ajustant à la bonne taille. Ensuite, l'animateur les invite à occuper l'espace avec différents types de marches à varier avec des stops, de variations de rythmes et de niveau, tout comme ils l'ont fait pendant les échauffements.

Lors d'un signal convenu, les participants s'arrêtent et tournent leur tête sans changer la position du corps, en regardant vers le public. Des mouvements de bras et de jambes, symétriques ou asymétriques peuvent être réalisés au cours du parcours par le biais de signaux différents (un battement de main, un mot clé, un son).

Dans un deuxième temps, l'intervenant demande aux étudiants de s'as- 
Apprentissage plurilingue et théâtre. Le chemin fructueux de la pluridisciplinarité

A. B. De SAnctis

DÉPLOYER LA COMMUNICATION À TRAVERS LES ARTS seoir sur une chaise, les bras posés sur les jambes, le regard vers le public, et de mimer, l'un après l'autre et de manière fluide, un état d'âme de leur choix. Les spectateurs interviennent et essayent de deviner les états joués par leurs collègues sur scène. Les deux groupes s'échangent le rôle, puis à la fin de l'exercice, ils expriment ce qu'ils ont ressenti et observé pendant la séquence sur le masque.

Du point de vue physiologique et psychologique, le port du masque agit sur le souffle et change la perception sensorielle de l'exécutant. Il cache le visage de ce dernier vis-à-vis du regard du public, lui transmettant une sensation de protection et l'aidant à déployer davantage son expression corporelle.

En remplacement du masque, dans cette partie, l'intervenant peut choisir de travailler avec des objets. Un des exercices consiste à accompagner les étudiants de manière graduelle afin qu'ils approchent premièrement l'objet de manière quotidienne (montrer aux autres comment utiliser un parapluie dans sa fonction ordinaire), puis de manière métaphorique (dévoiler aux autres comment un parapluie peut se transformer en une canne de promenade). En conclusion de l'exercice, les participants essaient de «devenir» corporellement l'objet à l'aide de la perception de ses formes, de ses couleurs et des matériaux avec lesquels il est fabriqué. Les objets, détachés progressivement de leur fonction quotidienne, aident les participants à mettre en relation des aires conceptuelles éloignées et à libérer et déployer leur imaginaire. Ce procédé leur permet de raconter une histoire à l'aide d'un support concret, tout en découvrant les potentialités du décalage de sens et de la multiplication des points de vue.

L'aller-retour entre significations réelles et vocabulaire imagé poussent l'exécutant à établir des connexions entre anciennes et nouvelles connaissances de manière aléatoire. Par l'action ces liens s'encrent dans le corps et aident l'exécutant à mémoriser le vocabulaire par la voie de la dissociation.

La quatrième étape de l'atelier s'articule autour de la création de la partition physique, dramaturgique et vocale. D'habitude, lorsqu'on propose un atelier de théâtre, les participants s'attendent à être rapidement confrontés à un texte à apprendre par cœur et à mettre en scène.

Cette manière de procéder ne correspond pas à la méthode employée dans cet atelier où l'on privilégie la contribution des participants (écriture et montage) à la présentation d'un texte préétabli (lecture et découpage). Multiples sont les matériaux qui peuvent être utilisés pour stimuler la rédaction d'un court texte, suivant les sujets et les domaines privilégiés par les participants. Si l'atelier se déroule sur une période de temps étenduc, les textes à produire peuvent être le fruit de traite- 
Apprentissage plurilingue et théâtre. Le chemin fructueux de la pluridisciplinarité

A. B. De SANCtis

\section{RÉVÉLER,}

DÉVELOPPER ET

PORTER SA VOIX:

DE L'ÉCOUTE À

L'ÉMISSION

GONSGIENTE ments plus complexes et plus longs, s'inspirant et croisant à la fois des sources iconographiques, littéraires, scientifiques, musicales, etc.

Dans le cas d'un atelier intensif, le matériel iconographique apparait comme le plus adapté à stimuler la potentialité créative des étudiants. L'intervenant dispose au sol d'images de toute sorte (issues de photos, des arts plastiques, de films, d'affiches, etc.), et demande aux participants d'en choisir une parmi celles soumises à leur attention. Quelques minutes plus tard, après avoir observé l'image retenue, chacun la décrit en quelques phrases à l'ensemble des participants disposés en cercle, en s'arrêtant sur quelques détails (couleurs, personnages, espace, lumière, libres associations, sensations etc.), et en mentionnant les éléments qui ont davantage retenu leur attention et motivé leur choix.

Une fois le premier tour terminé, la tâche se complexifie. Premièrement, les participants se mettent deux par deux et chacun examine l'image choisie par le partenaire. Deuxièmement, après avoir partagé les nouveaux éléments qu'ils ont repérés, ils fournissent au reste du groupe une description plus complète et articulée, fruit d'un travail (écrit ou oral) commun. Troisièmement, ils inventent et rédigent une histoire en mettant en relation les deux images, ensuite, ils la jouent en improvisant devant leurs camarades.

Les étudiants puisent leur inspiration dans leur univers personnel pour décrire l'image et rédiger le texte. De cette manière, ils relient leur propre imaginaire à la production linguistique, en s'appropriant des structures linguistiques et en les organisant eux-mêmes sur la base de leurs compétences et de leurs intérêts. Des liens affectifs entre les langues étrangères et le vécu des participants sont ainsi établis, et le parcours d'apprentissage accroit sa puissance en bénéficiant d'un enracinement fructueux. Les considérations de Francisco Varela confortent l'idée que les capacités d'apprentissage et de mémorisation sont liées à l'expérience personnelle. Il explique à ce propos: «Les facultés cognitives sont inextricablement liées à l'historique de ce qui est vécu, de la même manière qu'un sentier, au préalable inexistant, apparait en marchant» (Varela 1996: 111).

Lors de la cinquième étape de l'atelier, les participants entreprennent l'échauffement vocal basé sur l'exploration d'exercices qui les aide à maitriser leur respiration, à porter la voix et à utiliser des résonateurs différents pour affiner et élargir la gamme de sons à leur disposition.

En cercle, ils inspirent et expirent sur deux, puis quatre, puis huit temps. Ensuite, ils tapotent le visage avec les doigts, ils massent l'intérieur de la bouche et les gencives avec la langue. Des sons plus et moins graves sont émis par la bouche, par le nez, par le haut de la tête, par le front, par le ventre, etc; pour s'aider les étudiants placent 
Apprentissage plurilingue et théâtre. Le chemin fructueux de la pluridisciplinarité

A. B. De SAnctis

\section{POUR UNE \\ PERSONNALISATION DE L'APPRENTISSAGE}

une ou deux mains à la hauteur de chaque résonateur concerné. Ensuite, ils prononcent des mots ou des phrases quotidiennes en utilisant des tonalités et des volumes variés: en expirant, en articulant de manière exagérée, en rigolant, en pleurant, en chuchotant, etc. Enfin, à tour de rôle, chacun suggère aux camarades quelques formes différentes de projeter la voix.

Après avoir échauffé l'appareil vocal, chacun propose une phrase d'une chanson qu'il connaît par cœur et la répète par petits fragments jusque à ce que les autres camarades l'apprennent. Pour cet exercice, il est demandé de se servir uniquement de l'écoute et de la répétition pour intégrer la musique et les paroles de la phrase musicale partagée. Quand cette dernière a été apprise par le groupe, l'épreuve évolue et, un par un, les participants donnent des indications qui visent à nuancer l'émission vocale (exploration de la phrase musicale par une voix grave, aiguë, nasale, d'enfant, de vieux, d'un animal au choix, etc.). Enfin, les étudiants relient l'une après l'autre toutes les phrases proposées par l'ensemble afin de les façonner en une seule chanson à exécuter ensemble.

Lors de la sixième et demière étape de l'atelier, les étudiants disposent d'une demi-heure pour assembler la partition physique et textuelle, résultat du travail sur les images, en la combinant à leur convenance à la phrase musicale choisie et recréée à partir de l'exploration de différentes qualités de chant. Après la présentation finale des solos, les étudiants se réunissent en cercle afin d'établir un bilan de l'atelier.

La phase de montage qui clôture l'atelier demande aux participants un certain investissement dans le processus créatif et tend à les responsabiliser.

Ainsi, il leur est demandé de sélectionner eux-mêmes les matériaux, d'affiner le texte et les actions physiques et vocales, et d'effectuer des choix précis et définitifs en vue de leur création finale. Le chemin parcouru durant tout l'atelier, leur permet de s'approprier les deux langues de manière active et ludique et de transformer les connaissances linguistiques et culturelles implicites en connaissances explicites. Les étudiants se concentrent en priorité sur les nouvelles contraintes posées par les exercices de théâtre et, dans un premier temps, ce processus les oblige à ne plus porter leur attention sur les règles linguistiques, grâce à l'activation des filtres cognitifs. L'apprentissage croisé des deux langues se déroule ainsi de manière pragmatique, et déclenche un sentiment de soulagement chez l'étudiant lors de la prise de parole grâce à la suspension temporaire de jugement sur les critères grammaticaux et syntaxiques.

Le parcours proposé par cet atelier se fonde sur la conviction que les 
Apprentissage plurilingue et théâtre. Le chemin fructueux de la pluridisciplinarité

A. B. De SAnctis

\section{RÉFÉRENCES}

corps et les vécus des participants occupent une place fondamentale dans le processus d'apprentissage. Il propose en effet d'associer la pratique théâtrale et l'enseignement combiné de l'italien et du français pour favoriser l'assimilation de ces deux langues par l'action. Comme le rappelle Jauss:

«Dans la mesure où la jouissance esthétique libère de la contrainte pratique du travail et des besoins naturels de la vie quotidienne, elle fonde une fonction sociale spécifique par laquelle l'expérience esthétique s'est depuis toujours distinguée de toutes les autres activités. [...] L'expérience esthétique ne s'oppose aucunement par nature à la connaissance ni à l'action» (Jauss 1990: 141).

La compénétration entre arts et pédagogie ouvre à l'apprenant des chemins inattendus, fructueux et joyeux.

ADEN J. 2016. Créer, innover par le théâtre: pour une pédagogie enactive des langues, in I. Capron Puozzo (dir.), La créativité en éducation et en formation, Louvain-la-neuve, de Boeck supérieur.

BATESON G. 1977. Vers une écologie de l'esprit, vol. 1, Paris, Éditions du Seuil,

BERTHOZ A., PETIT J.-L. 2006. Phénoménologie et physiologie de l'action, Odile Jacob.

COHEN M. 1997. L'acquisition du langage par l'enfant, La surdité du premier âge, colloque international de Besançon, ed. Camponovo, 1969, p. 29, cité par Jean-Marie Pradier dans La scène et la fabrique des corps, Presses Universitaires de Bordeaux.

HALL E. T. 2014/1966. La dimension cachée, Essais, Points, JAUSS H. R. 1990 (1978). Pour une esthétique de la réception, Tel, Gallimard.

TELLIER M. 2012. Former à l'étude de la gestuelle: réflexion didactiques, pp. 73-85 in R. Vion, A. Giacomi, C. Vargas (dirs.), La corporalité du langage. Multimodalité, discours et écriture, PUP. 